\title{
Selective ring opening of silylated vinyloxiranes and reactivity of azido-alcohols
}

\author{
Jean-Charles Marié, Christine Courillon,* and Max Malacria* \\ Université Pierre et Marie Curie-Paris6, Laboratoire de Chimie Organique, UMR CNRS 7611, \\ Institut de Chimie Moléculaire FR2769, 04 place Jussieu, Tour 44-54 2ème étage, B229, F- \\ 75252 Paris cedex 05, France \\ E-mail: christine.courillon@paris7.jussieu.fr, max.malacria@upmc.fr
}

\section{Dedicated to Professor Lutz F Tietze on the occasion of his $65^{\text {th }}$ birthday}

\begin{abstract}
The chemoselective ring opening of silylated vinyloxiranes and of silylated epoxy alcohols by sodium azide has been studied. The reactivity of the $\alpha$-silylated azido-alcohols thus synthesized has been investigated and the formation of highly functionalized products such as acyl azides, azidovinylsilanes, and silylated aldehydes has been rationalized.
\end{abstract}

Keywords: Azide, chemoselectivity, oxirane, nucleophilic ring opening, rearrangement, silicon

\section{Introduction}

The discovery of new functionalized substrates remains an interesting challenge for organic chemists. In this respect, silylated azido-alcohols have a high degree of functionalization, and could be precursors for amino-alcohols which make efficient bidentate ligands for catalytic transformations. ${ }^{1}$ Our group has been developing the study of the reactivity of highly functionalized molecules such as silylated vinyloxiranes towards organometallic species, ${ }^{2}$ lithiated bases $^{3}$ and heterogeneous nucleophiles. ${ }^{4}$ These reactions have proved to be extremely stereo- and chemoselective, since the $\alpha, \beta$-epoxy- $\gamma, \delta$-unsaturated silanes 1a-c have three electrophilic centers and two acidic protons. However, the reactivity of compounds $\mathbf{1 a}-\mathbf{c}$ has shown that of the two groups attached to the oxirane, the silyl group has a greater effect than the vinyl moiety. Not only do deprotonations occur preferentially in the position $\alpha$ - to the vinyl functionality, as we previously reported, ${ }^{3,4}$ but also nucleophilic openings of the trans-oxirane occur preferentially with scission of the $\mathrm{C}-\mathrm{O}$ bond $\alpha-$ to the silicon atom rather than to the vinyl group. ${ }^{2,4}$ We should stress that the ring-opening of both cis- and trans- $\alpha, \beta$-epoxy- $\gamma, \delta$ unsaturated-silanes 1a-c, and of the silylated alcohols 2, 3, 4 (epoxy-protected or not) by 
heteroatomic nucleophiles such as azide anion or phenylsulfide lead mainly to substitution in the position $\alpha$ - to the silicon atom. Of particular interest and novelty is the behavior of the consecutively $\mathrm{S}_{\mathrm{N}} 2$ - formed azido-alcohols towards sulfonyl chlorides.

\section{Results and Discussion}

\section{$\mathrm{S}_{\mathrm{N}} 2$ ring opening of silylated oxiranes with hetero-nucleophiles}

The regio- and stereoselective ring opening ${ }^{5}$ of the silylated oxiranes by nitrogen- or sulfurnucleophiles is an interesting target in the field of organic synthesis. With the purpose of obtaining $\alpha$-azido-alcohols, we submitted compounds $\mathbf{1 a}-\mathbf{c}, \mathbf{2}, \mathbf{3}$, and $\mathbf{4}$ to sodium azide and ammonium chloride in aqueous methanol. ${ }^{6}$

Table 1. Ring opening of silylated oxiranes with $\mathrm{NaN}_{3}$

\begin{tabular}{|c|c|c|c|c|c|}
\hline TBL & $S, O$ & $\mathrm{NaN}$ & (5 equiv.) & TBDMS, & $\mathrm{OH}$ \\
\hline & $N$ & $\mathrm{NH}_{4}$ & I (2.5 equiv.) & & R \\
\hline & & $\mathrm{MeC}$ & $\mathrm{H} / \mathrm{H}_{2} \mathrm{O}, 55^{\circ} \mathrm{C}$ & & \\
\hline entry & $\mathrm{R}$ & & substrate & yield(\%) & product \\
\hline 1 & $(E) \mathrm{CH}=\mathrm{CO}_{2} \mathrm{M}$ & $\mathrm{Me}$ & trans-1a-(E) & 59 & 5 \\
\hline 2 & $(\mathrm{Z}) \mathrm{CH}=\mathrm{CO}_{2} \mathrm{M}$ & $\mathrm{Me}$ & trans-1a-(Z) & 0 & \\
\hline 3 & $(E) \mathrm{CH}=\mathrm{Ph}$ & & trans-1b-(E) & $0^{a}$ & \\
\hline 4 & $(\mathrm{Z}) \mathrm{CH}=\mathrm{Ph}$ & & trans $-1 b-(Z)$ & 13 & 6 \\
\hline 5 & $\mathrm{CH}=\mathrm{CH}_{2}$ & & trans-1c & $51^{b}$ & 7 \\
\hline 6 & $\mathrm{CH}=\mathrm{CH}_{2}$ & & cis-1c & $44^{\mathrm{c}}$ & 8 \\
\hline 7 & $\mathrm{CH}_{2} \mathrm{OH}$ & & 2 & 87 & 9 \\
\hline 8 & $\mathrm{CH}_{2} \mathrm{OTBDM}$ & & 3 & $71^{d}$ & 10 \\
\hline 9 & $\mathrm{CH}_{2} \mathrm{OCH}_{2} \mathrm{Pr}$ & & 4 & 70 & 11 \\
\hline & $\mathrm{HO}$ & & $12: \mathrm{R}=\mathrm{Cl}$ & $=\mathrm{CH}_{2}$ & \\
\hline & TBL & $\mathrm{N}_{3}$ & 13: $\mathrm{R}=\mathrm{CH}$ & OTBDMS & \\
\hline
\end{tabular}

${ }^{a}$ Total degradation of the starting material. ${ }^{b}$ Traces of compound $\mathbf{1 2}$ arising from the $\mathrm{S}_{\mathrm{N}} 2^{\prime}$ reaction of the azide anion on the vinyl-oxirane moiety are obtained in an inseparable mixture with the main product. ${ }^{\mathrm{c}}$ Secondary product $\mathbf{1 2}$ is isolated in $18 \%$ yield. ${ }^{\mathrm{d}}$ Compound $\mathbf{1 0}$ is the major regioisomer obtained, with the other $\mathrm{S}_{\mathrm{N}} 2$ adduct 13 in a 5.3/1 ratio.

Concerning the ring-opening of the silylated vinyl oxiranes 1a-c, Table 1 (entries 1-6) shows that only the azido-alcohols issued from the ring opening of the oxirane $\alpha-$ to the silicon atom are obtained in 13 to $59 \%$ yield depending on the substituent and the configuration of the alkene 
moiety. Compound (Z)- trans-1a was totally recovered under the reaction conditions (entry 2), whereas compound (E)-trans-1b degraded completely (entry 3). The best yield is achieved with the substrate (E)- trans-1a which is substituted by an electron-withdrawing ester group (entry 1). Compound cis-1c is transformed in an overall yield of $62 \%$, including $44 \%$ of the $\mathrm{S}_{\mathrm{N}} 2$ azido product and $18 \%$ of the diastereoselective $\mathrm{S}_{\mathrm{N}} 2$ ' adduct with the $E$ - configuration (entry 6). Degradation (entry 3) and low yield (entry 4) for phenyl derivatives trans-1b reflect the already reported high sensitivity of these compounds to acidic conditions. ${ }^{7}$ Other experimental conditions have been tested on compound 1c to improve this $\mathrm{S}_{\mathrm{N}} 2$ reaction. Different solvents such as DMF, DMSO or $\mathrm{CH}_{3} \mathrm{CN}$ gave the azido-alcohol 7 in lower yields than in aqueous methanol. Various nitrogen nucleophiles such as hydrazines ( $N$-phenyl-, $N, N$-dimethyl-, and unsubstituted hydrazine), trimethylsilyl azide or benzylamine did not transform compound 1c, which was recovered totally. The addition of Lewis acids was also useless.

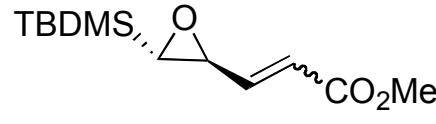

trans-1a-(E or Z)

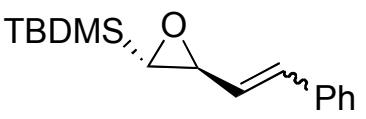

cis-1b-(E or Z)

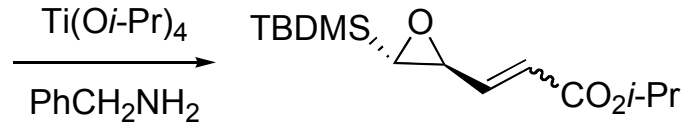

14-E : $95 \%$

$14-Z: 88 \%$
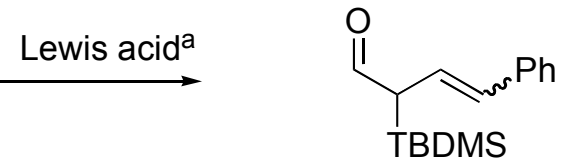

15-(E or Z): 45-98\%

Scheme 1. (a) $\mathrm{BF}_{3} \cdot \mathrm{Et}_{2} \mathrm{O}, \mathrm{ZnCl}_{2}, \mathrm{Ti}\left(\mathrm{OPr}_{i}\right)_{4}$ or $\mathrm{MgBr}_{2}$.

In the presence of Lewis acids $\left(\mathrm{BF}_{3} \cdot \mathrm{Et}_{2} \mathrm{O}, \mathrm{ZnCl}_{2}\right.$ or $\left.\mathrm{AlCl}_{3}\right)$ trimethylsilyl azide, potassium phthalimide or benzylamine led only to the degradation of compounds trans-1a- (E- or $Z$-). When titanium tetra-iso-propoxide is used, transesterification products 14- (E or $Z$ ) were isolated in good yields of $95 \%(\mathbf{1 4}-\boldsymbol{E})$ and $88 \%(\mathbf{1 4}-Z)$ (Scheme 1). The phenyl-substituted derivatives trans-1b $(E$ or $Z)$ rearranged into $\alpha$-silylated- $\beta, \gamma$-unsaturated-aldehydes 15- $(E$ or $Z$ ) in yields depending on the nature of the Lewis acid (Scheme 1). ${ }^{8}$

Concerning the ring-opening of the silylated epoxy alcohol $\mathbf{2}$ and silylated epoxy ethers $\mathbf{3}$ and 4, the azido-alcohols 9, 10, and 11 are prepared in better yields (entries 7-9, Table 1) than the vinyl analogs 1a-c. In the case of the disilylated substrate $\mathbf{3}$ (entry 8, Table 1), the major azidoalcohol 10 is formed in $60 \%$ yield but some ring-opening occurred $\beta$ - to the silicon atom, yielding also the regioisomer 13 in $11 \%$ yield. This latter result indicates that not only is the regioselectivity controlled by the silicon atom linked to the oxirane, but also it is influenced by the hydroxy group, as had been reported previously by Chakraborty. ${ }^{9,10}$ However, this lack of regioselectivity could be circumvented, since direct silylation of azido-diol 9 afforded compound 10 in $91 \%$ yield. 
The ring-opening of the diastereomers trans- and cis-1c by thiophenol in the presence of triethylamine in methanol occur efficiently within three hours. The $\mathrm{S}_{\mathrm{N}} 2$ reaction is less chemoselective than with sodium azide since the two regioisomeric silylated thio-alcohols are obtained. ${ }^{4}$

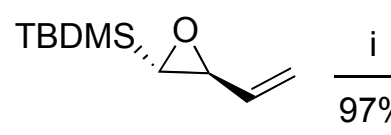

trans-1c

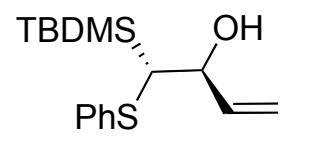

16

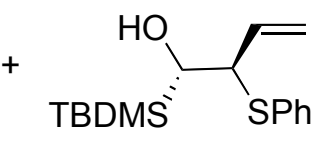

$6.8 / 1 \quad 17$

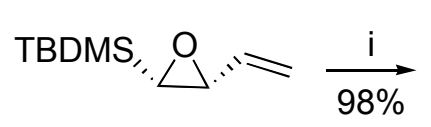

cis-1c

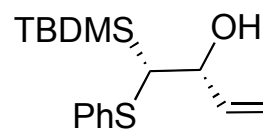

18

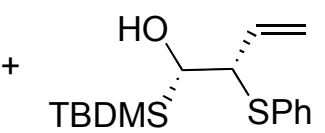

3.5/1 19

(i) $\mathrm{PhSH}$ (5 equiv.), $\mathrm{NEt}_{3}$ (2.5 equiv.), $\mathrm{MeOH}, 60^{\circ} \mathrm{C}, 3 \mathrm{~h}$.

\section{Scheme 2}

In the major adduct, the silicon and the sulfur atoms are both linked to the same carbon atom. Compound trans-1c is transformed into a 6.8/1 ratio of compounds $\mathbf{1 6}$ and $\mathbf{1 7}$ in a yield of $97 \%$ whereas cis-1c gives a 3.5/1 ratio of compounds 18 and 19 in a yield of 98\%. The minor thioalcohols 17 and 19 could be separated and isolated. The selectivity apparently depends on the relative configuration of the starting oxirane (Scheme 2).

Reaction of silylated oxiranes with nitrogen- and sulfur (in the case of vinyloxiranes)containing nucleophiles yields substituted alcohols, allylic or not, mainly arising from the opening of the oxirane in the position $\alpha$ - to the silicon atom. The $\alpha$-azido-alcohols listed in Table 1 were perfect substrates for Staudinger reductive cyclization into aziridines. ${ }^{11}$ However, none of these silylated azides could lead to an aziridine, and only degradation was observed. These results prompted us to investigate other aspects of the silylated azido-alcohols' reactivity, among which stands the activation of the hydroxyl group in the $\beta$-position to the silicon atom.

\section{Reactivity of silylated $\alpha$-azido-alcohols}

The activation of a secondary hydroxyl group as a sulfonate ester can proceed in a basic medium by reacting the alcohol with sulfonyl chloride derivatives.

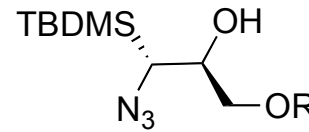

$10: R=$ OTBDMS

$11: \mathrm{R}=\mathrm{OBn}$

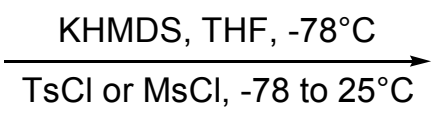

$20(85 \%): R=$ OTBDMS

\section{Scheme 3}


Tosyl- and mesyl- chlorides reacted on compounds $\mathbf{1 0}$ and $\mathbf{1 1}$ with potassium hexamethylsilylamide to yield respectively the vinyl azides 20 (85\%) and 21 (75\%) arising from the formal elimination of $t$-butyldimethylsilanol caused by a Peterson elimination reaction (Scheme 3). Although only one diastereomer is obtained, the $13.7 \mathrm{~Hz}$ coupling constant of the two vinylic protons in the ${ }^{1} \mathrm{H}-\mathrm{NMR}$ could not be attributed unambiguously to one configuration of the carbon-carbon double bond. However, NOE experiments have shown no effect between the vinylic protons and we could propose that the double bond has an $E$-configuration, indicating a syn-elimination mechanism which is classically observed for Peterson eliminations run in basic media.

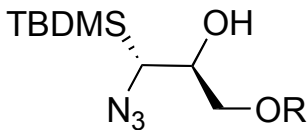

$10: R=O T B D M S$

$11: \mathrm{R}=\mathrm{OBn}$

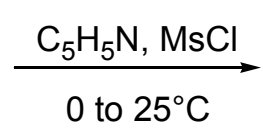

$22(43 \%): R=$ OTBDMS

$(E)-23(53 \%): \mathrm{R}=\mathrm{OBn}$

\section{Scheme 4}

The preparation of the sulfonate esters from azido-alcohols $\mathbf{1 0}$ and $\mathbf{1 1}$ was tried in pyridine as the solvent. When submitting the silylated azido-alcohols $\mathbf{1 0}$ and $\mathbf{1 1}$ to mesyl chloride in pyridine we could isolate azido-vinylsilanes 22 and 23 in moderate yields of 43 and 53\%, respectively (Scheme 4). In the case of the allylic benzyl ether 23, NOE irradiation experiments clearly showed a syn-relationship between the vinylic proton and the silylated substituent, which indicated that the double bond has the E-configuration. At this stage of our study, we could consider different possible dehydration mechanisms without being able to discriminate between E2, E1cb, or E1 pathways. However, we showed that the proton $\alpha$ - to the silicon atom is sufficiently acidic to be abstracted by pyridine.

The great reactivity of the sulfonate ester derivatives of the silylated azido-alcohols has lead us to consider forming them at low temperature and under the reaction conditions usually used for primary alcohols.

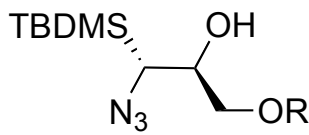

$10: R=O T B D M S$

$11: R=O B n$
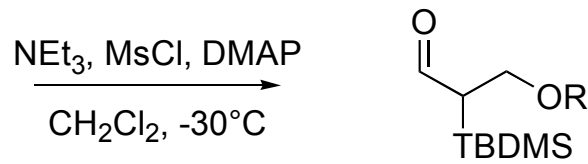

$26(46 \%): \mathrm{R}=$ OTBDMS

$27(37 \%): R=$ OBn, $11(26 \%)$

\section{Scheme 5}


Unexpectedly, the silylated azido-alcohols $\mathbf{1 0}$ and $\mathbf{1 1}$ are transformed into the $\alpha$-silylated aldehydes 26 and 27, respectively, in 46 and 37\% yield (Scheme 5). The formation of the aldehydes 26 and 27 could proceed via the hydrolysis either of the corresponding imines or of the $\alpha$-silylated-imide anion. The latter anionic intermediates have been reported to be formed by treatment of azido compounds in basic medium. The proton could be abstracted from a carbon atom bearing the azido substituent and an electron-withdrawing group which stabilizes the negative charge. Examples of this electron-deficient substituent being an ester group or a sulfone have been described in the literature. ${ }^{12-14}$ In our case, the silylated group could stabilize the negative charge $\alpha$ to the azido function and therefore could favor the formation of the imide anion. To investigate the importance of the azido group in this reaction, we prepared selectively the $\alpha$-benzylamino- $\alpha$-silylated alcohols $\mathbf{2 4}$ and $\mathbf{2 5}$ from the silylated epoxy-alcohol 2 . $^{15}$ Compound 25 was submitted to triethylamine, $N, N$-dimethylaminopyridine and mesyl chloride (Scheme 6).

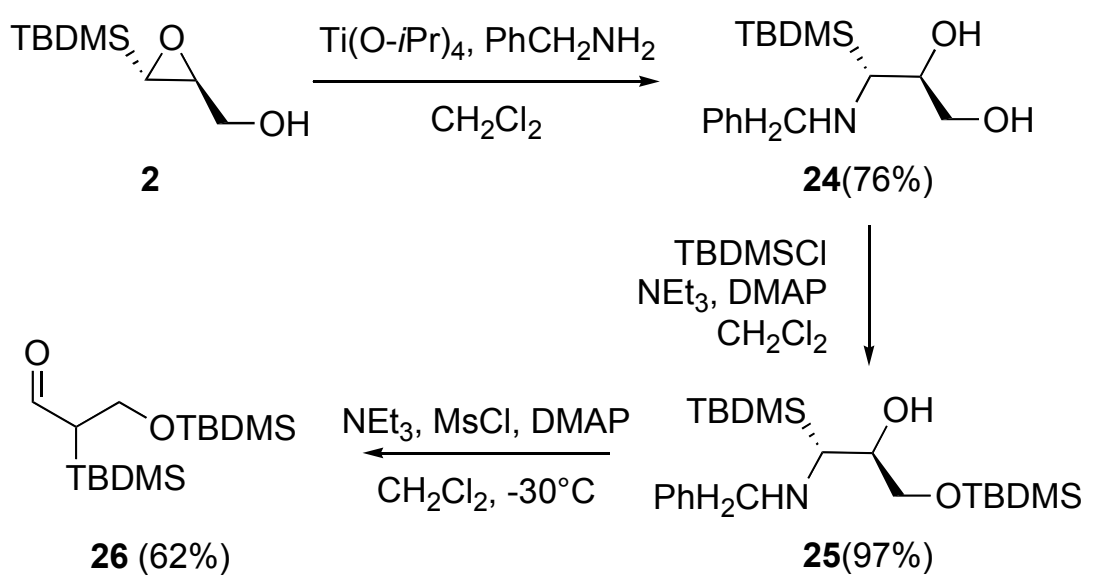

\section{Scheme 6}

The silicon-rearrangement yields the $\alpha$-silylated aldehyde 26, produced similarly from the azido-alcohol 10 (Scheme 5). We can propose a mechanism (Scheme 7) implying the activation of the amino alcohol $\mathbf{2 5}$ into the amino-mesylate $\mathbf{2 8}$ followed by the spontaneous migration of the silyl group together with elimination of the mesylate anion. The subsequent characterization of benzylamine and aldehyde $\mathbf{2 6}$ suggests the possible hydrolysis of the unisolated imine that could be obtained by transformation of intermediate 25 . 


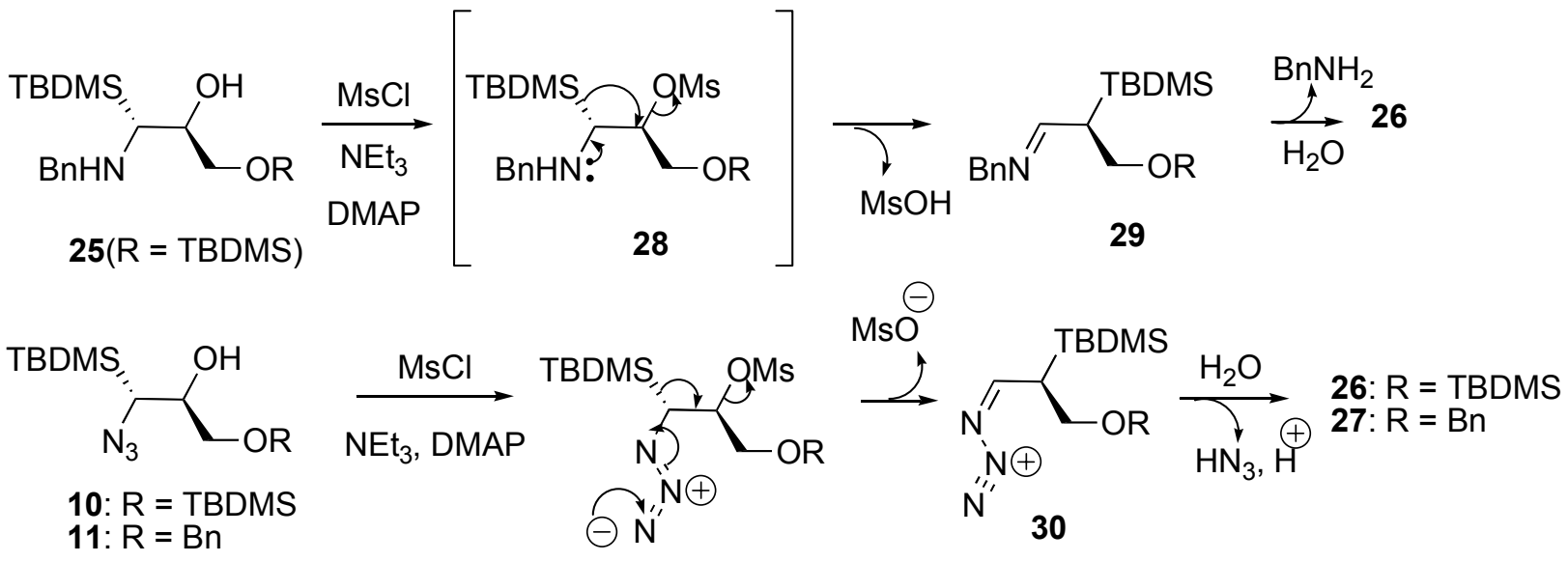

\section{Scheme 7}

A similar mechanism can be proposed for the azido-alcohols $\mathbf{1 0}$ and 11, in which the transposition/elimination step could yield the diazo-imine intermediate $\mathbf{3 0}$ which could react with water and eliminate hydrazoic acid to lead to the $\alpha$-silylated aldehyde (Scheme 7). To the best of our knowledge, this sila-pinacolic rearrangement of azido-alcohols has not previously been reported in the literature (Scheme 7).

\section{Conclusions}

We have investigated the nucleophilic ring-opening of silylated vinyloxiranes and of silylated epoxy alcohols with azide anion and conclude that the reaction is chemoselective and leads to the regioisomer in which the silicon atom and the azido group are $\alpha$ - to each other. The reactivity of the silylated azido-alcohols towards sulfonyl chlorides very much depends on the experimental conditions. We have shown that they can undergo Peterson elimination, dehydration, and silapinacolic rearrangement, leading respectively to acyl azides, azidovinylsilanes, and silylated aldehydes. The latter products are highly functionalized entities and are therefore interesting precursors for organic synthesis.

\section{Acknowledgements}

The authors thank the Ministry of Research for Jean-Charles Marié's PhD grant. Max Malacria is a member of the Institut Universitaire de France, and this institution is thanked for generous financial support of this work. 


\section{Experimental Section}

General Procedures. RT denotes room temperature; "petroleum" is the fraction having b.p. 40$60^{\circ} \mathrm{C}$.

Methyl 5-azido-5-(tert-butyldimethylsilyl)-4-hydroxy-pent-2-enoate (5). General procedure for ring-opening with azide ion. To a solution of the silylated vinyloxirane $(\boldsymbol{E})-\mathbf{1 a}(500 \mathrm{mg}$, $2.06 \mathrm{mmol}, 1.0$ equiv.) in $\mathrm{MeOH} / \mathrm{H}_{2} \mathrm{O}(8 / 1,5 \mathrm{~mL})$ were successively added, at $\mathrm{RT}, 671 \mathrm{mg}$ of $\mathrm{NaN}_{3}$ (10.31 mmol, 5.0 equiv.) and solid $\mathrm{NH}_{4} \mathrm{Cl}$ (276 mg, $5.16 \mathrm{mmol}, 2.5$ equiv.). The mixture was warmed to $55^{\circ} \mathrm{C}$ and stirred for $12-36 \mathrm{~h}$. It was then quenched with $10 \mathrm{~mL}$ of saturated aqueous $\mathrm{NaHCO}_{3}$ solution and the organic phase washed with brine. The combined organic layers were dried over $\mathrm{MgSO}_{4}$ and the solvents removed in vacuo after filtration. The crude product was purified by flash chromatography on silica gel (petroleum/ $\mathrm{Et}_{2} \mathrm{O}: 80 / 20$ ) affording the azido-alcohol 5 in $59 \%$ yield $\left(347 \mathrm{mg}, 1.22 \mathrm{mmol}\right.$ ) as a pale yellow oil. IR (neat) $\left(\mathrm{cm}^{-1}\right) 3470$, 2950, 2090, 1720, 1640, 1450, 1250, 840. ${ }^{1} \mathrm{H}-\mathrm{NMR}\left(400 \mathrm{MHz}, \mathrm{CDCl}_{3}\right) . \delta 6.97$ (dd, $1 \mathrm{H}, J=15.2$, $\left.5.6 \mathrm{~Hz}, \mathrm{H}_{3}\right), 6.11\left(\mathrm{dd}, 1 \mathrm{H}, J=15.2,1.5 \mathrm{~Hz}, \mathrm{H}_{2}\right), 4.55$ (bt, $\left.1 \mathrm{H}, J=4.1 \mathrm{~Hz}, \mathrm{H}_{4}\right), 3.73\left(\mathrm{~s}, 3 \mathrm{H}, \mathrm{H}_{9}\right)$, $3.28\left(\mathrm{~d}, 1 \mathrm{H}, J=4.1 \mathrm{~Hz}, \mathrm{H}_{5}\right), 3.91$ (bs, 1H, OH), 0.93 (s, 9H, $\left.\mathrm{H}_{7}\right), 0.07$ (s, 3H, $\left.\mathrm{H}_{6}\right), 0.03$ (s, 3H,

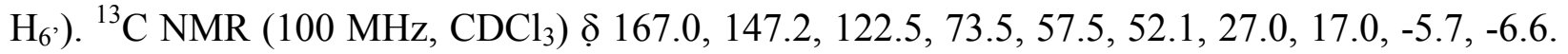
(Found: $\mathrm{C}, 50.62 ; \mathrm{H}, 8.41 ; \mathrm{N}, 15.10 . \mathrm{C}_{12} \mathrm{H}_{23} \mathrm{~N}_{3} \mathrm{O}_{3} \mathrm{Si}$ requires $\mathrm{C}, 50.50 ; \mathrm{H}, 8.12 ; \mathrm{N}, 14.72 \%$ ).

1-Azido-1-(t-butyldimethylsilyl)-4-phenyl-but-3-en-2-ol (6). Using the general procedure indicated for the preparation of the azido-ester $\mathbf{5}$, the azido-alcohol $\mathbf{6}$ was synthesized starting from silylated vinyloxirane $\mathbf{1 b}-(\boldsymbol{Z})(521 \mathrm{mg}, 2.0 \mathrm{mmol}$, 1 equiv.). After workup, the alcohol 6 was purified by flash chromatography on silica gel (petroleum/Et ${ }_{2} \mathrm{O}: 80 / 20$ ), affording a yellow oil in $13 \%$ yield $\left(79 \mathrm{mg}, 0.26 \mathrm{mmol}\right.$ ). IR (neat) $3380,2930,2090,1630,1250,830 \mathrm{~cm}^{-1} .{ }^{1} \mathrm{H}-$ NMR $\left(400 \mathrm{MHz}, \mathrm{CDCl}_{3}\right) \delta 7.35\left(\mathrm{~m}, 5 \mathrm{H}, \mathrm{H}_{6,7,8}\right), 6.64\left(\mathrm{~d}, 1 \mathrm{H}, J=11.6 \mathrm{~Hz}, \mathrm{H}_{4}\right), 5.90(\mathrm{dd}, 1 \mathrm{H}, J=$ 11.6, $\left.9.6 \mathrm{~Hz}, \mathrm{H}_{3}\right), 4.71\left(\mathrm{dd}, 1 \mathrm{H}, J=9.6,3.6 \mathrm{~Hz}, \mathrm{H}_{2}\right), 3.32\left(\mathrm{~d}, 1 \mathrm{H}, J=3.6 \mathrm{~Hz}, \mathrm{H}_{1}\right), 2.47(\mathrm{bs}, 1 \mathrm{H}$, $\mathrm{OH}), 0.84\left(\mathrm{~s}, 9 \mathrm{H}, \mathrm{H}_{10}\right), 0.03\left(\mathrm{~s}, 3 \mathrm{H}, \mathrm{H}_{9}\right),-0.27\left(\mathrm{~s}, 3 \mathrm{H}, \mathrm{H}_{9}\right) .{ }^{13} \mathrm{C}$ NMR $\left(100 \mathrm{MHz}, \mathrm{CDCl}_{3}\right) \delta$ 137.0, 134.1, 130.7, 129.5, 129.3, 128.5, 70.6, 59.0, 27.5, 17.4, -6.0, -6.3. Anal. Calcd for $\mathrm{C}_{16} \mathrm{H}_{25} \mathrm{~N}_{3} \mathrm{OSi}$ : C, 63.32; H, 8.30; N, 13.85. Found: C, 63.63; H, 8.60; N, 13.76.

1-Azido-1-tert-butyldimethylsilyl-but-3-en-2-ol (7). Following the general procedure given for the azido-alcohol 5, the trans-silylated vinyloxirane 1 c $(200 \mathrm{mg}, 1.08 \mathrm{mmol}, 1$ equiv.) gave 7 in $51 \%$ yield as a white solid (125 mg, $0.55 \mathrm{mmol})$, m.p. $61^{\circ} \mathrm{C}$. IR $\left(\mathrm{CHCl}_{3}\right) 3290,2930,2100,1650$, $1250,840 \mathrm{~cm}^{-1} .{ }^{1} \mathrm{H}$ NMR $\left(400 \mathrm{MHz}, \mathrm{CDCl}_{3}\right) \delta 5.99$ (ddd, $\left.1 \mathrm{H}, J=17.3,10.2,7.1 \mathrm{~Hz}, \mathrm{H}_{3}\right), 5.32$ $\left.\left(\mathrm{dt}, 1 \mathrm{H}, J=17.3,1.5 \mathrm{~Hz}, \mathrm{H}_{4}\right), 5.23\left(\mathrm{dt}, 1 \mathrm{H}, J=10.2,1.5 \mathrm{~Hz}, \mathrm{H}_{4}\right)^{\prime}\right), 4.35(\mathrm{dd}, 1 \mathrm{H}, J=7.1,4.1 \mathrm{~Hz}$, $\mathrm{H}_{2}$ ), 3.24 (d, $\left.1 \mathrm{H}, J=4.1 \mathrm{~Hz}, \mathrm{H}_{1}\right), 2.36$ (bs, 1H, OH), 0.92 (s, 9H, $\left.\mathrm{H}_{6}\right), 0.04$ (s, 3H, $\left.\mathrm{H}_{5}\right), 0.00$ (s, $\left.3 \mathrm{H}, \mathrm{H}_{5},\right) .{ }^{13} \mathrm{C} \mathrm{NMR}\left(100 \mathrm{MHz}, \mathrm{CDCl}_{3}\right) \delta 137.9,118.0,75.5,57.6,27.0,17.0,-5.9,-6.8\left(\mathrm{C}_{5},\right)$. Anal. Calcd for $\mathrm{C}_{10} \mathrm{H}_{21} \mathrm{~N}_{3} \mathrm{OSi}$ : C, 52.82; H, 9.31; N, 18.48. Found: C, 52.63; H, 9.15; N, 18.76\%. Traces of compound 12 were also detected.

1-Azido-1-tert-butyldimethylsilyl-but-3-en-2-ol (8). Following the general procedure given for the azido-alcohol $\mathbf{5}$, compound $\mathbf{8}$ was synthesized starting from the cis-silylated vinyloxirane 1c 
(200 mg, $1.08 \mathrm{mmol}, 1$ equiv.). After the usual workup and flash chromatography on silica gel (petroleum/Et ${ }_{2} \mathrm{O}: 80 / 20$ ), the azido-alcohol 8 was obtained in $44 \%$ yield as a colorless oil (109 $\mathrm{mg}, 0.48 \mathrm{mmol})$. IR (neat) 3280, 2950, 2100, 1660, 1250, $850 \mathrm{~cm}^{-1} .{ }^{1} \mathrm{H} \mathrm{NMR}\left(400 \mathrm{MHz}, \mathrm{CDCl}_{3}\right)$ $\delta 6.03\left(\mathrm{ddd}, 1 \mathrm{H}, J=17.3,10.2,5.6 \mathrm{~Hz}, \mathrm{H}_{3}\right), 5.35\left(\mathrm{dt}, 1 \mathrm{H}, J=17.3,1.0 \mathrm{~Hz}, \mathrm{H}_{4}\right), 5.24(\mathrm{dt}, 1 \mathrm{H}, J=$ 10.2, $\left.1.0 \mathrm{~Hz}, \mathrm{H}_{4}\right), 4.35$ (bs, 1H, $\left.\mathrm{H}_{2}\right), 2.88$ (d, 1H, J=3.0 Hz, H $\mathrm{H}_{1}, 2.12$ (bs, 1H, OH), 0.94 (s, 9H, $\left.\mathrm{H}_{6}\right), 0.15\left(\mathrm{~s}, 3 \mathrm{H}, \mathrm{H}_{5}\right), 0.10\left(\mathrm{~s}, 3 \mathrm{H}, \mathrm{H}_{5},\right) .{ }^{13} \mathrm{C} \mathrm{NMR}\left(100 \mathrm{MHz}, \mathrm{CDCl}_{3}\right) \delta 139.7,116.3,74.7,57.2$, 27.0, 17.0, -5.8, -6.2. Anal. Calcd for $\mathrm{C}_{10} \mathrm{H}_{21} \mathrm{~N}_{3} \mathrm{OSi}$ : C, 52.82; H, 9.31; N, 18.48; Found: C, $52.78 ; \mathrm{H}, 9.42 ; \mathrm{N}, 18.49 \%$. Compound 12 was also isolated in $18 \%$ yield (44 $\mathrm{mg}, 0.19 \mathrm{mmol}$ ) as a colorless oil.

(trans)-1-tert-Butyldimethylsilyl-4-azido-but-2-en-1-ol (12). IR (neat) 3300, 2940, 2090, 1670, 1240, $840 \mathrm{~cm}^{-1} .{ }^{1} \mathrm{H}$ NMR (400 MHz, $\left.\mathrm{CDCl}_{3}\right) \delta 5.99\left(\mathrm{dd}, 1 \mathrm{H}, J=15.3,5.1 \mathrm{~Hz}, \mathrm{H}_{2}\right), 5.61$ (dtd, $\left.1 \mathrm{H}, J=15.2,5.6,2.0 \mathrm{~Hz}, \mathrm{H}_{3}\right), 4.21\left(\mathrm{bd}, 1 \mathrm{H}, J=4.1 \mathrm{~Hz}, \mathrm{H}_{1}\right), 3.76\left(\mathrm{t}, 2 \mathrm{H}, J=5.6 \mathrm{~Hz}, \mathrm{H}_{4}\right), 0.95(\mathrm{~s}$, $\left.9 \mathrm{H}, \mathrm{H}_{6}\right), 0.01\left(\mathrm{~s}, 3 \mathrm{H}, \mathrm{H}_{5}\right),-0.03\left(\mathrm{~s}, 3 \mathrm{H}, \mathrm{H}_{5},\right) .{ }^{13} \mathrm{C} \mathrm{NMR}\left(100 \mathrm{MHz}, \mathrm{CDCl}_{3}\right) \delta$ 139.3, 118.6, 66.6, 53.0, 27.2, 17.3, -7.2, -8.9.

(trans)-Isopropyl 5-tert-butyldimethylsilyl-4,5-epoxypent-2-enoate ((Z)-14). To a cooled $\left(0^{\circ} \mathrm{C}\right)$ solution of the silylated vinyloxirane (Z)-1a $(363 \mathrm{mg}, 1.5 \mathrm{mmol}, 1$ equiv.) and benzylamine $(0.49 \mathrm{~mL}, 4.5 \mathrm{mmol}, 3$ equiv. $)$ in $\mathrm{CH}_{2} \mathrm{Cl}_{2}(3 \mathrm{~mL})$ was added titanium tetraisopropoxide $(0.90 \mathrm{~mL}, 3 \mathrm{mmol}, 2$ equiv.). The reaction mixture was stirred for $1 \mathrm{~h}$ at $\mathrm{RT}$ then heated at reflux for $12 \mathrm{~h}$. It was then quenched at RT with a satd aq. $\mathrm{Na}_{2} \mathrm{SO}_{4}(10 \mathrm{~mL})$ and filtered through a Celite pad. The mixture was diluted with AcOEt $(30 \mathrm{~mL})$, washed with brine, and dried over $\mathrm{Na}_{2} \mathrm{SO}_{4}$. The solvents were removed in vacuo and the crude product purified by flash chromatography (petroleum/Et $\left.{ }_{2} \mathrm{O}: 90 / 10\right)$, yielding 14-(Z) as a colorless oil (357 $\mathrm{mg}, 1.32 \mathrm{mmol}$, $88 \%$ ). IR (neat) 2930, 1720, 1650, 1420, 1250, 850 $\mathrm{cm}^{-1} .{ }^{1} \mathrm{H}$ NMR $\left(400 \mathrm{MHz}, \mathrm{CDCl}_{3}\right) \delta 5.87$ (d, $\left.1 \mathrm{H}, J=11.7 \mathrm{~Hz}, \mathrm{H}_{2}\right), 5.68\left(\mathrm{dd}, 1 \mathrm{H}, J=11.7,8.2 \mathrm{~Hz}, \mathrm{H}_{3}\right), 5.04\left(\mathrm{~h}, 1 \mathrm{H}, J=6.6 \mathrm{~Hz}, \mathrm{H}_{9}\right), 4.40$ (dd, $\left.1 \mathrm{H}, J=8.1,3.6 \mathrm{~Hz}, \mathrm{H}_{4}\right), 2.20\left(\mathrm{~d}, 1 \mathrm{H}, J=3.6 \mathrm{~Hz}, \mathrm{H}_{5}\right), 1.24\left(\mathrm{~d}, 6 \mathrm{H}, J=6.6 \mathrm{~Hz}, \mathrm{H}_{10}\right), 0.93(\mathrm{~s}, 9 \mathrm{H}$, $\left.\mathrm{H}_{7}\right), 0.01\left(\mathrm{~s}, 3 \mathrm{H}, \mathrm{H}_{6}\right),-0.02\left(\mathrm{~s}, 3 \mathrm{H}, \mathrm{H}_{6}\right) .{ }^{13} \mathrm{C} \mathrm{NMR}\left(100 \mathrm{MHz}, \mathrm{CDCl}_{3}\right) \delta 165.8,148.8,123.7$, 68.0, 51.3, 50.8, 26.8, 22.2, 17.0, -8.0. Anal. Calcd for $\mathrm{C}_{14} \mathrm{H}_{26} \mathrm{O}_{3} \mathrm{Si}$ : C, 62.18; H, 9.69; Found. C, $62.21 ; \mathrm{H}, 9.71 \%$.

(trans)-Isopropyl 5-tert-butyldimethylsilyl-4,5-epoxypent-2-enoate ((E)-14). Following the procedure for the preparation of 14-(Z), starting from the silylated vinyloxirane 1a-(E) (363 mg, $1.5 \mathrm{mmol}, 1$ equiv.), the title compound $\mathbf{1 4}-(\boldsymbol{E})$ was obtained in $95 \%$ yield (385 $\mathrm{mg}, 1.43 \mathrm{mmol})$ as a colorless oil. IR (neat) 2960, 1730, 1670, 1430, 1250, $830 \mathrm{~cm}^{-1} .{ }^{1} \mathrm{H} \mathrm{NMR}\left(400 \mathrm{MHz}, \mathrm{CDCl}_{3}\right)$ $\delta 6.54\left(\mathrm{dd}, 1 \mathrm{H}, J=15.2,7.6 \mathrm{~Hz}, \mathrm{H}_{3}\right), 6.10\left(\mathrm{~d}, 1 \mathrm{H}, J=15.3 \mathrm{~Hz}, \mathrm{H}_{2}\right), 5.02\left(\mathrm{~h}, 1 \mathrm{H}, J=6.1 \mathrm{~Hz}, \mathrm{H}_{9}\right)$, $3.21\left(\mathrm{dd}, 1 \mathrm{H}, J=7.6,3.1 \mathrm{~Hz}, \mathrm{H}_{4}\right), 2.25\left(\mathrm{~d}, 1 \mathrm{H}, J=3.6 \mathrm{~Hz}, \mathrm{H}_{5}\right), 1.22\left(\mathrm{~d}, 6 \mathrm{H}, J=6.1 \mathrm{~Hz}, \mathrm{H}_{10}\right)$, $0.91\left(\mathrm{~s}, 9 \mathrm{H}, \mathrm{H}_{7}\right),-0.01\left(\mathrm{~s}, 3 \mathrm{H}, \mathrm{H}_{6}\right),-0.08\left(\mathrm{~s}, 3 \mathrm{H}, \mathrm{H}_{6}\right)$ ). ${ }^{13} \mathrm{C} \mathrm{NMR}\left(100 \mathrm{MHz}, \mathrm{CDCl}_{3}\right) \delta 165.5$, 147.0, 124.1, 68.2, 53.8, 52.5, 26.7, 22.1, 17.0, -7.8, -8.2. Anal. Calcd for $\mathrm{C}_{14} \mathrm{H}_{26} \mathrm{O}_{3} \mathrm{Si}$ : C, 62.18; H, 9.69. Found: C, 62.19; H, 9.78. 


\section{1-tert-Butyldimethylsilyl-1-phenylsulfanyl-but-3-en-2-ol (16). General procedure for ring- opening with thiophenol}

To a solution of the silylated vinyloxirane $1 \mathrm{c}(100 \mathrm{mg}, 0.54 \mathrm{mmol}, 1$ equiv.) in $\mathrm{MeOH}(5 \mathrm{~mL})$ were successively added, at RT, $\mathrm{NEt}_{3}(0.15 \mathrm{~mL}, 1.08 \mathrm{mmol}, 2$ equiv.) and $\mathrm{PhSH}(0.28 \mathrm{~mL}, 2.71$ mmol, 5 equiv.). The reaction mixture was stirred for $1 \mathrm{~h}$ and then warmed at $60^{\circ} \mathrm{C}$ for 3 hours. After cooling down to RT, the medium was diluted with $25 \mathrm{~mL}$ of $\mathrm{CH}_{2} \mathrm{Cl}_{2}$, and the organic phase treated with sat. aq. $\mathrm{NH}_{4} \mathrm{Cl}$ solution. The aqueous layer was extracted with $\mathrm{CH}_{2} \mathrm{Cl}_{2}$, washed with brine and dried over $\mathrm{Na}_{2} \mathrm{SO}_{4}$. After filtration and removal of the solvents in vacuo, the residue was purified and the two isomers $\mathbf{1 6}$ and $\mathbf{1 7}$ could be separated by flash chromatography on silica gel (petroleum/ $\mathrm{CH}_{2} \mathrm{Cl}_{2}: 80 / 20$ ), affording colorless oils in a total yield of $97 \%$ (major, 16, 135 mg, $0.46 \mathrm{mmol}$; minor, 17, $20 \mathrm{mg}, 0.07 \mathrm{mmol}$ ). IR (neat) 3440, 3040, 2900, 1670, 1240, $820 \mathrm{~cm}^{-}$ ${ }^{1} .{ }^{1} \mathrm{H}$ NMR (400 MHz, $\left.\mathrm{CDCl}_{3}\right) \delta$ 7.50-7.18 (m, 5H, H9,10,11), 6.01 (ddd, 1H, J=17.3, 10.7, 6.1 $\left.\mathrm{Hz}, \mathrm{H}_{3}\right), 5.30\left(\mathrm{dd}, 1 \mathrm{H}, J=17.3,1.5 \mathrm{~Hz}, \mathrm{H}_{4}\right), 5.22\left(\mathrm{dd}, 1 \mathrm{H}, J=10.7,1.5 \mathrm{~Hz}, \mathrm{H}_{4}\right), 4.52(\mathrm{~m}, 1 \mathrm{H}$, $\left.\mathrm{H}_{2}\right), 3.04\left(\mathrm{~d}, 1 \mathrm{H}, J=3.0 \mathrm{~Hz}, \mathrm{H}_{1}\right), 2.46$ (s, 1H, OH), 0.99 (s, 9H, H ), 0.18 (s, 3H, H5), 0.18 (s, $3 \mathrm{H}, \mathrm{H}_{5}$ ). ${ }^{13} \mathrm{C} \mathrm{NMR}\left(100 \mathrm{MHz}, \mathrm{CDCl}_{3}\right) \delta 139.3,137.9,129.2,126.2,116.3,74.4,39.9,27.4$, 17.6, -4.5, -5.6. Exact mass: $m / z$ calcd for $\mathrm{C}_{16} \mathrm{H}_{27} \mathrm{OSSi}\left(\mathrm{MH}^{+}\right)$295.1552, found 295.1548

1-tert-Butyldimethylsilyl-2-phenylsulfanyl-but-3-en-1-ol (17). IR (neat) 3450, 3060, 2910, 1660, 1250, $840 \mathrm{~cm}^{-1} .{ }^{1} \mathrm{H}$ NMR (400 MHz, $\left.\mathrm{CDCl}_{3}\right) \delta 7.41-7.27\left(\mathrm{~m}, 5 \mathrm{H}, \mathrm{H}_{9,10,11}\right), 5.98(\mathrm{dt}, 1 \mathrm{H}, J$ $\left.=17.3,9.7 \mathrm{~Hz}, \mathrm{H}_{3}\right), 5.13\left(\mathrm{~d}, 1 \mathrm{H}, J=16.2, \mathrm{H}_{4}\right), 5.13\left(\mathrm{~d}, 1 \mathrm{H}, J=11.7, \mathrm{H}_{4}\right), 3.97(\mathrm{dd}, 1 \mathrm{H}, J=9.7$, $\left.2.5 \mathrm{~Hz}, \mathrm{H}_{2}\right), 3.56\left(\mathrm{~d}, 1 \mathrm{H}, J=2.5 \mathrm{~Hz}, \mathrm{H}_{1}\right), 2.17(\mathrm{bs}, 1 \mathrm{H}, \mathrm{OH}), 0.85\left(\mathrm{~s}, 9 \mathrm{H}, \mathrm{H}_{6}\right), 0.06\left(\mathrm{~s}, 3 \mathrm{H}, \mathrm{H}_{5}\right)$, $0.04\left(\mathrm{~s}, 3 \mathrm{H}, \mathrm{H}_{5}\right) .{ }^{13} \mathrm{C} \mathrm{NMR}\left(100 \mathrm{MHz}, \mathrm{CDCl}_{3}\right) \delta 134.8,133.9,133.8,129.3,128.1,118.7,62.7$, 59.4, 27.0, 17.2, $-6.7,-7.3$.

1-tert-Butyldimethylsilyl-1-phenylsulfanyl-but-3-en-2-ol (18). Following the same procedure as for the preparation of 16, the allylic alcohol $\mathbf{1 8}$ was synthesized from the silylated vinyloxirane 1c (100 mg, $0.54 \mathrm{mmol}, 1$ equiv.), affording a mixture of two isomers 18 and 19 (ratio 3.5/1, total 98\%) which was purified and separated (major 18, $121 \mathrm{mg}, 0.41 \mathrm{mmol}$; minor, $19,35 \mathrm{mg}, 0.12 \mathrm{mmol}$ ) by flash chromatography on silica gel (petroleum/ $\mathrm{CH}_{2} \mathrm{Cl}_{2}: 80 / 20$ ). IR (neat) $3420,3060,2920,1650,1240,820 \mathrm{~cm}^{-1} .{ }^{1} \mathrm{H}$ NMR $\left(400 \mathrm{MHz}, \mathrm{CDCl}_{3}\right) \delta 7.38-7.18(\mathrm{~m}, 5 \mathrm{H}$, $\left.\mathrm{H}_{9,10,11}\right), 5.79\left(\mathrm{ddd}, 1 \mathrm{H}, J=17.3,10.7,5.6 \mathrm{~Hz}, \mathrm{H}_{3}\right), 5.22\left(\mathrm{~d}, 1 \mathrm{H}, J=17.3 \mathrm{~Hz}, \mathrm{H}_{4}\right), 4.94(\mathrm{~d}, 1 \mathrm{H}, J$ $\left.=10.2 \mathrm{~Hz}, \mathrm{H}_{4}\right), 4.46\left(\mathrm{~m}, 1 \mathrm{H}, \mathrm{H}_{2}\right), 2.93\left(\mathrm{~d}, 1 \mathrm{H}, J=2.6 \mathrm{~Hz}, \mathrm{H}_{1}\right), 2.41(\mathrm{~d}, 1 \mathrm{H}, J=8.1 \mathrm{~Hz}, \mathrm{OH})$, $1.04\left(\mathrm{~s}, 9 \mathrm{H}, \mathrm{H}_{6}\right), 0.22\left(\mathrm{~s}, 3 \mathrm{H}, \mathrm{H}_{5}\right), 0.22\left(\mathrm{~s}, 3 \mathrm{H}, \mathrm{H}_{5}\right) .{ }^{13} \mathrm{C} \mathrm{NMR}\left(100 \mathrm{MHz}, \mathrm{CDCl}_{3}\right) \delta 140.7,138.2$, 129.3, 128.9, 126.1, 114.8, 73.3, 39.0, 27.5, 17.7, -5.3, -5.7. Exact mass: calcd for $\mathrm{C}_{16} \mathrm{H}_{27} \mathrm{OSSi}$ $\left(\mathrm{MH}^{+}\right)$295.1552, found 295.1557

1-tert-Butyldimethylsilyl-2-phenylsulfanyl-but-3-en-1-ol (19). IR (neat) 3480, 3070, 2960, $1620,1250,850 \mathrm{~cm}^{-1} .{ }^{1} \mathrm{H}$ NMR $\left(400 \mathrm{MHz}, \mathrm{CDCl}_{3}\right) \delta 7.30-7.26\left(\mathrm{~m}, 5 \mathrm{H}, \mathrm{H}_{9,10,11}\right), 5.68(\mathrm{dt}, 1 \mathrm{H}, J$ $\left.=17.3,9.6 \mathrm{~Hz}, \mathrm{H}_{3}\right), 5.01\left(\mathrm{dd}, 1 \mathrm{H}, J=9.7,1.5 \mathrm{~Hz}, \mathrm{H}_{4}\right), 4.95\left(\mathrm{~d}, 1 \mathrm{H}, J=16.3, \mathrm{H}_{4}\right), 3.71(\mathrm{dd}, 1 \mathrm{H}, J$ $\left.=9.6,9.2 \mathrm{~Hz}, \mathrm{H}_{2}\right), 3.37\left(\mathrm{dd}, 1 \mathrm{H}, J=9.2,2.6 \mathrm{~Hz}, \mathrm{H}_{1}\right), 2.56(\mathrm{~d}, 1 \mathrm{H}, J=2.5 \mathrm{~Hz}, \mathrm{OH}), 0.96(\mathrm{~s}, 9 \mathrm{H}$, $\left.\mathrm{H}_{6}\right), 0.06\left(\mathrm{~s}, 3 \mathrm{H}, \mathrm{H}_{5}\right), 0.06\left(\mathrm{~s}, 3 \mathrm{H}, \mathrm{H}_{5},\right) .{ }^{13} \mathrm{C} \mathrm{NMR}\left(100 \mathrm{MHz}, \mathrm{CDCl}_{3}\right) \delta 135.3,132.9-124.5$, 115.3, 63.4, 60.8, 26.0, 16.3, -6.0, -8.9. 


\section{(E)-3-tert-Butyldimethylsilyl-1-tert-butyldimethylsilanyloxy-prop-2-ene (31) (Scheme 8)}

To a cooled $\left(0^{\circ} \mathrm{C}\right)$ solution of silylated allylic alcohol $\mathbf{3 2}^{16}(1.72 \mathrm{~g}, 10.0 \mathrm{mmol}, 1.0 \mathrm{equiv}$. $)$ in $\mathrm{CH}_{2} \mathrm{Cl}_{2}(10 \mathrm{~mL})$ were successively added $\mathrm{NEt}_{3}(2.78 \mathrm{~mL}, 20.0 \mathrm{mmol}, 2.0$ equiv.), DMAP (122 $\mathrm{mg}, 1.0 \mathrm{mmol}, 0.1$ equiv.) and, after 5 minutes stirring at $0^{\circ} \mathrm{C}$, $\mathrm{TBSCl}(1.66 \mathrm{~g}, 11.0 \mathrm{mmol}, 1.1$ equiv.). The reaction mixture was allowed to warm up to RT and after stirring for 30 minutes was diluted with $40 \mathrm{~mL}$ of $\mathrm{CH}_{2} \mathrm{Cl}_{2}$. The medium was quenched with saturated aq. $\mathrm{NH}_{4} \mathrm{Cl}$ solution, washed with brine, and the combined organic layers dried over $\mathrm{Na}_{2} \mathrm{SO}_{4}$. After filtration and evaporation of the solvent, the crude product was purified by flash chromatography on silica gel (petroleum/ $\mathrm{Et}_{2} \mathrm{O}: 95 / 5$ ) affording the disilylated compound 31 as a white solid, in 93\% yield (2.66 g, $9.28 \mathrm{mmol})$, m.p. $=55^{\circ} \mathrm{C}$; IR $\left(\mathrm{CH}_{2} \mathrm{Cl}_{2}\right) 2950,1670,1250,840 \mathrm{~cm}^{-1} .{ }^{1} \mathrm{H} \mathrm{NMR}(200 \mathrm{MHz}$, $\left.\mathrm{CDCl}_{3}\right) \delta 6.14\left(\mathrm{dt}, 1 \mathrm{H}, J=18.8,3.6 \mathrm{~Hz}, \mathrm{H}_{2}\right), 5.94\left(\mathrm{~d}, 1 \mathrm{H}, J=18.8, \mathrm{H}_{3}\right), 4.24\left(\mathrm{~d}, 2 \mathrm{H}, J=3.6, \mathrm{H}_{1}\right)$, $0.95\left(\mathrm{~s}, 9 \mathrm{H}, \mathrm{H}_{5}\right), 0.90\left(\mathrm{~s}, 9 \mathrm{H}, \mathrm{H}_{8}\right), 0.10\left(\mathrm{~s}, 6 \mathrm{H}, \mathrm{H}_{7}\right), 0.05\left(\mathrm{~s}, 6 \mathrm{H}_{1} \mathrm{H}_{4}\right) .{ }^{13} \mathrm{C} \mathrm{NMR}\left(50 \mathrm{MHz}, \mathrm{CDCl}_{3}\right)$ $\delta 146.9,125.6,66.3,26.8,26.3,18.8,16.9,-4.8$, -5.8. Anal. Calcd for $\mathrm{C}_{15} \mathrm{H}_{34} \mathrm{OSi}_{2}$ : $\mathrm{C}, 62.86 ; \mathrm{H}$, 11.96. Found: C, 62.74; H, 12.08 .

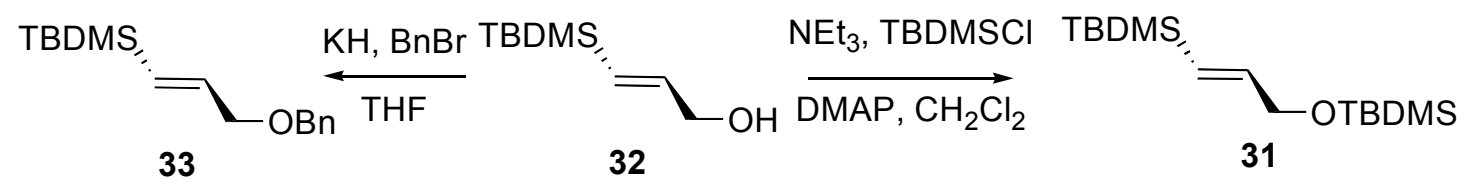

\section{Scheme 8}

(trans)-3-tert-Butyldimethylsilyl-2,3-epoxy-1-tert-butyldimethylsilanyloxypropane (3)To a cooled $\left(0^{\circ} \mathrm{C}\right)$ solution of the vinylsilane 31 (1.00 g, $3.49 \mathrm{mmol}, 1$ equiv.) in $\mathrm{CH}_{2} \mathrm{Cl}_{2}(40 \mathrm{~mL})$ was slowly added $7.40 \mathrm{~g}$ of $m$-CPBA (70\% with water and 3-chlorobenzoic acid, $30.03 \mathrm{mmol}, 1.5$ equiv.). The reaction mixture was allowed to warm up to RT and after stirring for $2 \mathrm{~h}, 20 \mathrm{~mL}$ of brine was added. The organic layer was then dried over $\mathrm{MgSO}_{4}$ and after partial evaporation of the solvent, $10 \mathrm{~mL}$ of pentane was added. The precipitate was then filtered off using a short pad of Celite. This operation was repeated four times to assure complete removal of the $m$-CPBA and the corresponding acid. The residue was then purified by flash chromatography on silica gel (petroleum/AcOEt:80/20), affording the silylated oxirane 3 as a white solid in $96 \%$ yield (1.01 g, $3.35 \mathrm{mmol})$.

Starting from the silylated epoxy-alcohol 2 (471 mg, $2.5 \mathrm{mmol}, 1$ equiv.), the silylation of the hydroxyl group was achieved following the procedure described for the preparation of the disilylated compound 31. Compound 3 was obtained in 91\% yield (688 mg, $2.28 \mathrm{mmol}$ ). m.p. $95^{\circ} \mathrm{C}$; IR $\left(\mathrm{CH}_{2} \mathrm{Cl}_{2}\right) 2960,1250,850 \mathrm{~cm}^{-1} .{ }^{1} \mathrm{H} \mathrm{NMR}\left(400 \mathrm{MHz}, \mathrm{CDCl}_{3}\right) \delta 3.84(\mathrm{dd}, 1 \mathrm{H}, J=11.6$, $\left.3.5 \mathrm{~Hz}, \mathrm{H}_{1}\right), 3.66\left(\mathrm{dd}, 1 \mathrm{H}, J=11.6,5.2 \mathrm{~Hz}, \mathrm{H}_{1}\right.$ ) $), 2.96$ (ddd, $\left.1 \mathrm{H}, J=5.6,3.6,3.5 \mathrm{~Hz}, \mathrm{H}_{2}\right), 2.17$ (d, $\left.1 \mathrm{H}, J=3.6 \mathrm{~Hz}, \mathrm{H}_{3}\right), 0.97\left(\mathrm{~s}, 9 \mathrm{H}, \mathrm{H}_{5}\right), 0.92$ (s, 9H, $\left.\mathrm{H}_{8}\right), 0.10$ (s, 3H, H7), 0.09 (s, 3H, H${ }_{7}$ ), 0.04 (s, $\left.3 \mathrm{H}, \mathrm{H}_{4}\right),-0.04\left(\mathrm{~s}, 3 \mathrm{H}, \mathrm{H}_{4}\right)$ ). ${ }^{13} \mathrm{C} \mathrm{NMR}\left(100 \mathrm{MHz}, \mathrm{CDCl}_{3}\right) \delta 65.9,55.8,46.7,26.6,25.9,18.4$, 16.7, -5.2, -5.3, -8.1, -8.5. Anal. Calcd for $\mathrm{C}_{15} \mathrm{H}_{34} \mathrm{O}_{2} \mathrm{Si}_{2}$ : C, 59.54; H, 11.33. Found: C, 59.34; $\mathrm{H}$, $11.52 \%$. 
(E)-3-tert-Butyldimethylsilyl-1-benzyloxy-prop-2-ene (33) (Scheme 8). To a cooled $\left(0^{\circ} \mathrm{C}\right)$ suspension of KH (30\% in mineral oil, $1.60 \mathrm{~g}, 12.0 \mathrm{mmol}, 1.2$ equiv.) in dry THF (60 mL), was added dropwise a solution of the allylic alcohol 32 (1.72 g, $10.0 \mathrm{mmol}, 1.0$ equiv.) in THF (20 $\mathrm{mL}$ ). After 15 minutes at $0^{\circ} \mathrm{C}$, the medium was allowed to warm up to RT for an additional 1 hour, and benzyl bromide (1.67 mL, $14.0 \mathrm{mmol}, 1.4$ equiv.) was slowly added to the mixture. After stirring for 12 hours, the mixture was quenched with $30 \mathrm{~mL}$ of a sat. aq. $\mathrm{NH}_{4} \mathrm{Cl}$ solution. The organic phase was washed with brine and the combined organic layers dried over $\mathrm{MgSO}_{4}$. After filtration and evaporation of the solvent, the crude product was purified by flash chromatography on silica gel (petroleum/ $\mathrm{Et}_{2} \mathrm{O}: 95 / 5$ ), affording the benzylic ether $\mathbf{3 3}$ as a colorless oil (2.38 g, $9.10 \mathrm{mmol}$.) in 91\% yield. IR (neat) $3050,2920,1670,1240,820 \mathrm{~cm}^{-1}{ }^{1} \mathrm{H}$ NMR (400 MHz, $\left.\mathrm{CDCl}_{3}\right) \delta 7.40-7.32\left(\mathrm{~m}, 5 \mathrm{H}, \mathrm{H}_{\mathrm{Ar}}\right), 6.19\left(\mathrm{dt}, 1 \mathrm{H}, J=18.8,5.1 \mathrm{~Hz}, \mathrm{H}_{2}\right) 5.99$ (d, $\left.1 \mathrm{H}, J=18.8 \mathrm{~Hz}, \mathrm{H}_{3}\right), 4.57\left(\mathrm{~s}, 2 \mathrm{H}, \mathrm{H}_{7}\right), 4.12\left(\mathrm{~d}, 2 \mathrm{H}, J=5.1 \mathrm{~Hz}, \mathrm{H}_{1}\right), 0.93\left(\mathrm{~s}, 9 \mathrm{H}, \mathrm{H}_{5}\right), 0.08(\mathrm{~s}, 6 \mathrm{H}$, $\left.\mathrm{H}_{4}\right) .{ }^{13} \mathrm{C} \mathrm{NMR}\left(100 \mathrm{MHz}, \mathrm{CDCl}_{3}\right) \delta 144.0,138.6,129.7,128.7-128.1,127.9,73.5,72.5,26.7$, 16.8, -5.9. Anal. Calcd for $\mathrm{C}_{16} \mathrm{H}_{26} \mathrm{OSi}$; C, 73.22; H, 9.98. Found: C, 73.06; H, 10.07\%.

trans-3-tert-Butyldimethylsilyl-2,3-epoxy-1-benzyloxypropane (4). To a cooled $\left(0^{\circ} \mathrm{C}\right)$ solution of the silylated allylic benzyl ether 33 (1.31 g, $5.0 \mathrm{mmol}, 1$ equiv.) in $\mathrm{CH}_{2} \mathrm{Cl}_{2}$ (40 mL) was slowly added $7.40 \mathrm{~g}$ of $m$-CPBA (70\% in water; 3-chlorobenzoic acid, $30.03 \mathrm{mmol}, 1.5$ equiv.). The reaction mixture was allowed to warm up to RT, and after stirring for $2 \mathrm{~h}, 20 \mathrm{~mL}$ of brine was added to the medium. The organic layer was then dried over $\mathrm{MgSO}_{4}$ and after partial evaporation of the solvent, $10 \mathrm{~mL}$ of pentane was added. The precipitate was then filtered off, using a short pad of Celite. This operation was repeated four times to assure complete removal of the $m$-CPBA and the corresponding acid. The residue was then purified by flash chromatography on silica gel (petroleum/AcOEt:80/20), affording compound 4 in $96 \%$ yield as a colorless oil (1.34 g, $4.8 \mathrm{mmol})$. IR (neat) 3010, 2950, 1250, $830 \mathrm{~cm}^{-1} .{ }^{1} \mathrm{H}$ NMR $\left(400 \mathrm{MHz}, \mathrm{CDCl}_{3}\right) \delta 7.38-$ $7.32\left(\mathrm{~m}, 5 \mathrm{H}, \mathrm{H}_{\mathrm{Ar}}\right), 4.67\left(\mathrm{~d}, 1 \mathrm{H}, J=11.7 \mathrm{~Hz}, \mathrm{H}_{7}\right), 4.59\left(\mathrm{~d}, 1 \mathrm{H}, J=12.2 \mathrm{~Hz}, \mathrm{H}_{7}\right), 3.87(\mathrm{dd}, 1 \mathrm{H}, J=$ 11.2, $\left.2.6 \mathrm{~Hz}, \mathrm{H}_{1}\right), 3.41\left(\mathrm{dd}, 1 \mathrm{H}, J=11.2,6.1 \mathrm{~Hz}, \mathrm{H}_{1}\right.$ ), 3.09 (ddd, 1H, $J=6.1,3.1,2.0 \mathrm{~Hz}, \mathrm{H}_{2}$ ), $2.18\left(\mathrm{~d}, 1 \mathrm{H}, J=2.0 \mathrm{~Hz}, \mathrm{H}_{3}\right), 1.00\left(\mathrm{~s}, 9 \mathrm{H}, \mathrm{H}_{5}\right), 0.05\left(\mathrm{~s}, 3 \mathrm{H}, \mathrm{H}_{4}\right),-0.01\left(\mathrm{~s}, 3 \mathrm{H}_{,} \mathrm{H}_{4}\right) .{ }^{13} \mathrm{C}$ NMR $(100$ $\left.\mathrm{MHz}, \mathrm{CDCl}_{3}\right) \delta 138.2,128.7-128.0,128.5,73.4,72.8,54.5,46.3,26.8,16.9,-8.0$, -8.2. Anal. Calcd for $\mathrm{C}_{16} \mathrm{H}_{26} \mathrm{O}_{2} \mathrm{Si}$ : C, 69.01; H, 9.41; Found: C, 68.93; H, 9.42\%.

3-Azido-3-tert-butyldimethylsilyl-propan-1.2-diol (9). Using the general procedure as for the preparation of azidoester 5, the azido-diol $\mathbf{9}$ was synthesized starting from the epoxy-alcohol 2 (188 mg, $1.00 \mathrm{mmol}, 1$ equiv.) in $87 \%$ yield as a colorless oil (201 $\mathrm{mg}, 0.87 \mathrm{mmol})$. IR (neat) 3380, 2950, 2100, 1250, $840 \mathrm{~cm}^{-1} .{ }^{1} \mathrm{H}$ NMR $\left(400 \mathrm{MHz}, \mathrm{CDCl}_{3}\right) \delta 3.92\left(\mathrm{~m}, 1 \mathrm{H}, \mathrm{H}_{2}\right), 3.69(\mathrm{~m}, 1 \mathrm{H}$, $\left.\mathrm{H}_{1}\right), 3.27\left(\mathrm{~d}, 1 \mathrm{H}, J=5.1 \mathrm{~Hz}, \mathrm{H}_{3}\right), 0.96\left(\mathrm{~s}, 9 \mathrm{H}, \mathrm{H}_{5}\right), 0.10\left(\mathrm{~s}, 3 \mathrm{H}, \mathrm{H}_{4}\right), 0.07\left(\mathrm{~s}, 3 \mathrm{H}, \mathrm{H}_{4}\right) .{ }^{13} \mathrm{C} \mathrm{NMR}$ $\left(100 \mathrm{MHz}, \mathrm{CDCl}_{3}\right) \delta 73.5,64.3,55.5,26.8,16.8,-6.2,-6.7$.

\section{3-Azido-3-tert-butyldimethylsilyl-1-tert-butyldimethylsinalyloxy-propan-2-ol (10).}

Following the general procedure given for the preparation of the azido-ester 5, the title compound was synthesized starting from the silylated oxirane 3 (605 mg, $2.00 \mathrm{mmol}$, 1 equiv.), in $71 \%$ global yield (491 mg, $1.42 \mathrm{mmol}$ ) as a mixture of two isomers $\mathbf{1 0}$ (413 $\mathrm{mg}, 1.19 \mathrm{mmol}$ ) and 13 (78 mg, $0.22 \mathrm{mmol}$ ) which could be separated by flash chromatography on silica gel 
(petroleum $\left./ \mathrm{CH}_{2} \mathrm{Cl}_{2}: 80 / 20\right)$. Starting from the azido-diol 9 (347 $\mathrm{mg}, 1.50 \mathrm{mmol}, 1$ equiv.), the selective silylation of the primary hydroxyl group was released following the procedure for the preparation of the disilylated compound 31. The azido-alcohol $\mathbf{1 0}$ was obtained in $91 \%$ yield as a colorless oil (472 mg, $1.36 \mathrm{mmol}$ ). IR (neat) 3350, 2950, 2090, 1230, $830 \mathrm{~cm}^{-1}$. ${ }^{1} \mathrm{H}$ NMR (400 $\left.\mathrm{MHz} \mathrm{CDCl}_{3}\right) \delta 3.85\left(\mathrm{~m}, 1 \mathrm{H}, \mathrm{H}_{2}\right), 3.70\left(\mathrm{~d}, 2 \mathrm{H}, J=5.4 \mathrm{~Hz}, \mathrm{H}_{1}\right), 3.21\left(\mathrm{~d}, 1 \mathrm{H}, J=6.2 \mathrm{~Hz}, \mathrm{H}_{3}\right), 2.76$ (d, 1H, $J=3.6 \mathrm{~Hz}, \mathrm{OH}), 0.97$ (s, 9H, $\left.\mathrm{H}_{5}\right), 0.93$ (s, 9H, H8), 0.12 (s, 3H, H7), 0.11 (s, 3H, H $\mathrm{H}_{7}$ ), $0.10\left(\mathrm{~s}, 3 \mathrm{H}, \mathrm{H}_{4}\right), 0.08\left(\mathrm{~s}, 3 \mathrm{H}, \mathrm{H}_{4}\right.$ ). ${ }^{13} \mathrm{C} \mathrm{NMR}\left(100 \mathrm{MHz}, \mathrm{CDCl}_{3}\right) \delta 73.1,64.5,53.8,26.9,26.0$, 18.4, 17.0, -5.3, -5.9, -6.6. Anal. Calcd for $\mathrm{C}_{15} \mathrm{H}_{35} \mathrm{~N}_{3} \mathrm{O}_{2} \mathrm{Si}_{2}: \mathrm{C}, 52.13 ; \mathrm{H}, 10.21 ; \mathrm{N}, 12.16$. Found: C, 51.95; H, 10.25; N, 11.89\%.

2-Azido-3-tert-butyldimethylsilyl-1-tert-butyldimethylsinalyloxy-propan-3-ol (13). IR (neat) 3380, 2940, 2110, 1250, $850 \mathrm{~cm}^{-1} .{ }^{1} \mathrm{H}$ NMR (400 MHz, $\left.\mathrm{CDCl}_{3}\right) \delta 4.02$ (ddd, $1 \mathrm{H}, J=8.0,3.6,2.8$ $\left.\mathrm{Hz}, \mathrm{H}_{2}\right), 3.75\left(\mathrm{dd}, 1 \mathrm{H}, J=11.2,8.0 \mathrm{~Hz}, \mathrm{H}_{1}\right), 3.53\left(\mathrm{bd}, 1 \mathrm{H}, J=10.6 \mathrm{~Hz}, \mathrm{H}_{1}\right), 3.31(\mathrm{~d}, 1 \mathrm{H}, J=2.8$ $\left.\mathrm{Hz}, \mathrm{H}_{3}\right), 1.97$ (bs, 1H, OH), 0.96 (s, 18H, H5,8), 0.16 (s, 3H, H $), 0.15$ (s, 3H, H $\left.{ }_{7}\right), 0.07$ (s, 3H, $\left.\mathrm{H}_{4}\right), 0.04\left(\mathrm{~s}, 3 \mathrm{H}, \mathrm{H}_{4}\right) .{ }^{13} \mathrm{C} \mathrm{NMR}\left(100 \mathrm{MHz}, \mathrm{CDCl}_{3}\right) \delta 75.5,64.1,57.1,26.7,25.9,18.1,16.7$, 4.1, -4.6, -6.5, -7.1. Anal. Calcd for $\mathrm{C}_{15} \mathrm{H}_{35} \mathrm{~N}_{3} \mathrm{O}_{2} \mathrm{Si}_{2}$ : C, 52.13; H, 10.21; N, 12.16. Found: C, 52.29; H, 10.37; N, $11.88 \%$.

3-Azido-3-tert-butyldimethylsilyl-1-benzyloxy-propan-2-ol (11). The procedure was exactly the same as described for the synthesis of 5, starting from the silylated oxirane 4 (278 $\mathrm{mg}, 1.0$ mmol, 1 equiv.). The azido-alcohol 11 was obtained in $70 \%$ yield as a colorless oil (225 $\mathrm{mg}, 0.7$ mmol). IR (neat) 3450, 3010, 2930, 2090, 1250, $840 \mathrm{~cm}^{-1}{ }^{1} \mathrm{H}$ NMR (400 MHz, $\mathrm{CDCl}_{3}$ ) $\delta 7.36-$ $7.31\left(\mathrm{~m}, 5 \mathrm{H}, \mathrm{H}_{\mathrm{Ar}}\right), 4.60\left(\mathrm{~d}, 1 \mathrm{H}, J=11.7 \mathrm{~Hz}, \mathrm{H}_{7}\right), 4.55\left(\mathrm{~d}, 1 \mathrm{H}, J=11.7 \mathrm{~Hz}, \mathrm{H}_{7}\right), 4.01(\mathrm{ddd}, 1 \mathrm{H}, J$ $\left.=7.1,5.6,3.6 \mathrm{~Hz}, \mathrm{H}_{2}\right), 3.60\left(\mathrm{dd}, 1 \mathrm{H}, J=9.6,7.1 \mathrm{~Hz}, \mathrm{H}_{1}\right), 3.56\left(\mathrm{dd}, 1 \mathrm{H}, J=9.6,3.6 \mathrm{~Hz}, \mathrm{H}_{1}\right.$ ) ), $3.26\left(\mathrm{~d}, 1 \mathrm{H}, J=5.6 \mathrm{~Hz}, \mathrm{H}_{3}\right), 0.94\left(\mathrm{~s}, 9 \mathrm{H}, \mathrm{H}_{5}\right), 0.05\left(\mathrm{~s}, 3 \mathrm{H}, \mathrm{H}_{4}\right), 0.01\left(\mathrm{~s}, 3 \mathrm{H}, \mathrm{H}_{4}\right) .{ }^{13} \mathrm{C} \mathrm{NMR}(100$ $\left.\mathrm{MHz}, \mathrm{CDCl}_{3}\right) \delta 137.9,128.9,128.3,128.2,73.8,72.4,72.0,54.8,27.1,17.2,-5.8,-6.5$. Anal. Calcd for $\mathrm{C}_{16} \mathrm{H}_{27} \mathrm{~N}_{3} \mathrm{O}_{2} \mathrm{Si}$ : C, 59.78; H, 8.47; N, 13.07. Found: C, 60.13; H, 8.73; N, 12.95\%

\section{(E) 1-Azido-3-tert-butyldimethylsilanyloxy-prop-1-ene (20)}

To a cooled $\left(-78^{\circ} \mathrm{C}\right)$ solution of the azido-alcohol $10(346 \mathrm{mg}, 1.0 \mathrm{mmol}, 1.00$ equiv.) in THF $(10 \mathrm{~mL})$ was slowly added KHMDS $(0.5 \mathrm{M}$ in toluene, $2.1 \mathrm{~mL}, 1.05 \mathrm{mmol}, 1.05$ equiv.). The medium was stirred for 10 minutes and mesyl chloride $(85 \mu \mathrm{L}, 1.10 \mathrm{mmol}, 1.10$ equiv.) was added rapidly. The reaction mixture was warmed up to RT and then quenched in water $(5 \mathrm{~mL})$. The organic layer was washed with brine and dried over $\mathrm{Na}_{2} \mathrm{SO}_{4}$. After filtration and removal of the solvents in vacuo, a crude product was obtained which was purified by flash chromatography on silica gel (petroleum/ $\mathrm{Et}_{2} \mathrm{O}: 98 / 2$ ), affording the vinyl azide $\mathbf{2 0}$ as a pale yellow oil (181 $\mathrm{mg}$, $0.85 \mathrm{mmol}$ ) in $85 \%$ yield. IR (neat) $2930,2100,1660,1250,830 \mathrm{~cm}^{-1} .{ }^{1} \mathrm{H} \mathrm{NMR}(400 \mathrm{MHz}$, $\left.\mathrm{CDCl}_{3}\right) \delta 6.11\left(\mathrm{~d}, 1 \mathrm{H}, J=13.8 \mathrm{~Hz}, \mathrm{H}_{1}\right), 5.49\left(\mathrm{dt}, 1 \mathrm{H}, J=13.7,5.6 \mathrm{~Hz}, \mathrm{H}_{2}\right), 4.20$ (dd, $2 \mathrm{H}, J=5.6$, $\left.1.5 \mathrm{~Hz}, \mathrm{H}_{3}\right), 0.92\left(\mathrm{~s}, 9 \mathrm{H}, \mathrm{H}_{5}\right), 0.09\left(\mathrm{~s}, 6 \mathrm{H}, \mathrm{H}_{4}\right) .{ }^{13} \mathrm{C} \mathrm{NMR}\left(100 \mathrm{MHz}, \mathrm{CDCl}_{3}\right) \delta 128.1,119.3,61.4$, 26.2, 18.7, -5.0

Anal. Calcd for $\mathrm{C}_{9} \mathrm{H}_{19} \mathrm{~N}_{3} \mathrm{OSi}$ : C, 50.67; H, 8.98; N, 19.70. Found: C, 50.41; H, 8.76; N, $19.95 \%$. 
(E) 1-Azido-3-benzyloxy-prop-1-ene (21). The procedure was as described previously, starting from the azido-alcohol 4 (161 $\mathrm{mg}, 0.50 \mathrm{mmol}, 1$ equiv.). The vinyl azide 21 was obtained as a yellow oil in $75 \%$ yield $\left(71 \mathrm{mg}, 0.37 \mathrm{mmol}\right.$ ). IR (neat) $3030,2850,2100,1650 \mathrm{~cm}^{-1} .{ }^{1} \mathrm{H} \mathrm{NMR}$ $\left(400 \mathrm{MHz}, \mathrm{C}_{6} \mathrm{D}_{6}\right) \delta 7.34$ (d, 2H, $\left.J=8.3 \mathrm{~Hz}, \mathrm{H}_{6}\right), 7.29$ (t, 2H, 8.4 Hz, H ), 7.21 (t, 1H, J= $7.8 \mathrm{~Hz}$, $\left.\mathrm{H}_{8}\right), 5.71\left(\mathrm{~d}, 1 \mathrm{H}, J=13.7 \mathrm{~Hz}, \mathrm{H}_{1}\right), 5.37\left(\mathrm{dt}, 1 \mathrm{H}, J=13.7,6.3 \mathrm{~Hz}, \mathrm{H}_{2}\right), 4.33\left(\mathrm{~s}, 2 \mathrm{H}, \mathrm{H}_{4}\right), 3.71(\mathrm{~d}$, $\left.2 \mathrm{H}, J=6.3 \mathrm{~Hz}, \mathrm{H}_{3}\right) .{ }^{13} \mathrm{C} \mathrm{NMR}\left(100 \mathrm{MHz}, \mathrm{C}_{6} \mathrm{D}_{6}\right) \delta 138.6\left(\mathrm{C}_{5}\right), 129.6\left(\mathrm{C}_{1}\right), 128.4\left(\mathrm{C}_{7}\right), 127.6$ $\left(\mathrm{C}_{6,8}\right), 116.2,71.8,67.4$.

\section{1-Azido-1-tert-butyldimethylsilyl-3-tert-butyldimethylsilyloxy-prop-1-ene (22)}

To a cooled $\left(0^{\circ} \mathrm{C}\right)$ solution of the azido-alcohol $10(173 \mathrm{mg}, 0.50 \mathrm{mmol}, 1.00$ equiv. $)$ in pyridine ( $2 \mathrm{~mL}$ ) was added mesyl chloride $\left(48 \mu \mathrm{L}, 0.63 \mathrm{mmol}, 1.25\right.$ equiv.). After 1 hour at $0^{\circ} \mathrm{C}$, the medium was warmed up to $25^{\circ} \mathrm{C}$ and stirred overnight. The reaction mixture was then diluted in $\mathrm{CH}_{2} \mathrm{Cl}_{2}(20 \mathrm{~mL})$ and $10 \mathrm{~mL}$ of a saturated aqueous $\mathrm{NH}_{4} \mathrm{Cl}$ solution was added under vigorous stirring. The organic phase was washed with brine and dried over $\mathrm{Na}_{2} \mathrm{SO}_{4}$. After filtration and removal of the solvent, the crude product was purified by flash chromatography on silica gel (petroleum/ $\left.\mathrm{Et}_{2} \mathrm{O}: 95 / 5\right)$, affording compound 22 as a colorless oil in $43 \%$ yield $(71 \mathrm{mg}, 0.21$ mmol). IR (neat) 2980, 2100, 1660, 1250, $840 \mathrm{~cm}^{-1} .{ }^{1} \mathrm{H} \mathrm{NMR}\left(400 \mathrm{MHz}, \mathrm{CDCl}_{3}\right) \delta 6.23(\mathrm{~s}, 1 \mathrm{H}$, $\left.\mathrm{H}_{2}\right), 4.27\left(\mathrm{~d}, 2 \mathrm{H}, J=1.0 \mathrm{~Hz}, \mathrm{H}_{3}\right), 0.89\left(\mathrm{~s}, 18 \mathrm{H}, \mathrm{H}_{5,8}\right), 0.08\left(\mathrm{~s}, 6 \mathrm{H}, \mathrm{H}_{4}\right), 0.05\left(\mathrm{~s}, 6 \mathrm{H}_{,} \mathrm{H}_{7}\right) .{ }^{13} \mathrm{C} \mathrm{NMR}$ $\left(100 \mathrm{MHz} \mathrm{CDCl}_{3}\right) \delta 132.1,128.0,59.2,26.0,25.0,17.4,16.2,-6.1,-6.4$. Anal. Calcd for $\mathrm{C}_{15} \mathrm{H}_{33} \mathrm{~N}_{3} \mathrm{OSi}_{2}$ : C, 54.99; H, 10.15; N, 12.83. Found: C, 55.02; H, 9.92; N, 12.85\%.

(E) 1-Azido-1-tert-butyldimethylsilyl-3-benzyloxy-prop-1-ene (2). Following the procedure described for the preparation of the compound 22, the silylated vinyl azide $\mathbf{2 3}$ was synthesized as a colorless oil (64 mg, $0.21 \mathrm{mmol}$; 53\% yield), starting from the azido-alcohol 11 (128 mg, 0.40 mmol, 1 equiv.). IR (neat) 3080, 2950, 2100, 1590, 1250, $830 \mathrm{~cm}^{-1} .{ }^{1} \mathrm{H} \mathrm{NMR}\left(400 \mathrm{MHz}, \mathrm{CDCl}_{3}\right)$ $\delta 7.24\left(\mathrm{~m}, 5 \mathrm{H}, \mathrm{H}_{9,10,11}\right), 6.29\left(\mathrm{~s}, 1 \mathrm{H}, \mathrm{H}_{2}\right), 4.36\left(\mathrm{~s}, 2 \mathrm{H}, \mathrm{H}_{7}\right), 4.03\left(\mathrm{~s}, 2 \mathrm{H}, \mathrm{H}_{3}\right), 0.80\left(\mathrm{~s}, 9 \mathrm{H}, \mathrm{H}_{5}\right), 0.00$ $\left(\mathrm{s}, 6 \mathrm{H}, \mathrm{H}_{4}\right) .{ }^{13} \mathrm{C} \mathrm{NMR}\left(100 \mathrm{MHz}, \mathrm{CDCl}_{3}\right) \delta 138.8,135.9,128.6,128.3,127.9,126.1,73.1,67.2$, 27.3, 17.7, -5.1. Anal. Calcd for $\mathrm{C}_{16} \mathrm{H}_{25} \mathrm{~N}_{3} \mathrm{OSi}$ : C, 63.32; H, 8.30; N, 13.85. Found: C, 63.38; $\mathrm{H}$, $8.08 ; \mathrm{N}, 14.01 \%$.

\section{2-tert-Butyldimethylsilyl-3-tert-butyldimethylsilanyloxypropanal (26)}

To a cooled $\left(-30^{\circ} \mathrm{C}\right)$ solution of the azido-alcohol 10 (1.38 g, $4.0 \mathrm{mmol}, 1.0$ equiv.) in $\mathrm{CH}_{2} \mathrm{Cl}_{2}$ $(16 \mathrm{~mL})$ were successively added $\mathrm{NEt}_{3}(0.67 \mathrm{~mL}, 4.8 \mathrm{mmol}, 1.2$ equiv.), DMAP (49 mg, 0.4 mmol, 0.1 equiv.) and, after 10 minutes, $\mathrm{MsCl}(0.34 \mathrm{~mL}, 4.4 \mathrm{mmol}, 1.1$ equiv.). After 1 hour, the reaction mixture was quenched in $10 \mathrm{~mL}$ of water and was warmed slowly to $\mathrm{RT}$. The organic phase was washed with brine and dried over $\mathrm{Na}_{2} \mathrm{SO}_{4}$. After removal of the solvent, the crude product was purified by flash chromatography on silica gel (petroleum/ $\mathrm{Et}_{2} \mathrm{O}: 90 / 10$ ), affording the aldehyde 26 as a colorless oil (557 mg, $1.84 \mathrm{mmol}, 46 \%$ yield). IR (neat) 2940, 1710, 1250, $840 \mathrm{~cm}^{-1} .{ }^{1} \mathrm{H}$ NMR $\left(400 \mathrm{MHz}, \mathrm{CDCl}_{3}\right) \delta 9.68\left(\mathrm{~d}, 1 \mathrm{H}, J=4.1 \mathrm{~Hz}, \mathrm{H}_{1}\right), 4.21(\mathrm{t}, 1 \mathrm{H}, J=10.2 \mathrm{~Hz}$, $\left.\mathrm{H}_{3}\right), 3.81\left(\mathrm{dd}, 1 \mathrm{H}, J=10.7,3.6 \mathrm{~Hz}, \mathrm{H}_{3}\right.$ ), 2.67 (dt, 1H, 10.2, 3.6 Hz, H $), 0.90\left(\mathrm{~s}, 9 \mathrm{H}_{1} \mathrm{H}_{5}\right), 0.84$ (s, $\left.9 \mathrm{H}, \mathrm{H}_{8}\right), 0.05$ (s, 3H, H4), $0.03\left(\mathrm{~s}, 3 \mathrm{H}, \mathrm{H}_{4}\right), 0.02$ (bs, 6H, H, $\left.\mathrm{H}_{7}\right) .{ }^{13} \mathrm{C}$ NMR $\left(100 \mathrm{MHz}, \mathrm{C}_{6} \mathrm{D}_{6}\right) \delta$ 200.8, 60.0, 50.9, 26.6, 25.9, 18.3, 17.4, -5.4, -6.3, -6.5. Anal. Calcd for $\mathrm{C}_{15} \mathrm{H}_{34} \mathrm{O}_{2} \mathrm{Si}_{2}: \mathrm{C}$, 59.54; H, 11.33. Found: C, 59.61; H, 11.56\%. 
2-tert-Butyldimethylsilyl-3-benzyloxypropanal (27). Following the same procedure as described for the preparation of the aldehyde $\mathbf{2 6}$, the $\alpha$-silylated aldehyde $\mathbf{2 7}$ was synthesized starting from the azido-alcohol 11 (524 mg, $1.63 \mathrm{mmol}, 1$ equiv.) in 37\% yield (168 $\mathrm{mg}, 0.6$ $\mathrm{mmol}$ ). Some starting material was recovered (136 mg, $0.42 \mathrm{mmol}, 26 \%$ ). IR (neat) 3010, 2930, 1700, 1250, $820 \mathrm{~cm}^{-1} .{ }^{1} \mathrm{H}$ NMR $\left(400 \mathrm{MHz}, \mathrm{CDCl}_{3}\right) \delta 9.75\left(\mathrm{~d}, 1 \mathrm{H}, J=3.7 \mathrm{~Hz}, \mathrm{H}_{1}\right), 7.37-7.29(\mathrm{~m}$, $\left.5 \mathrm{H}, \mathrm{H}_{9,10,11}\right), 4.52\left(\mathrm{~s}, 2 \mathrm{H}, \mathrm{H}_{7}\right), 4.14\left(\mathrm{t}, 1 \mathrm{H}, J=10.2 \mathrm{~Hz}, \mathrm{H}_{3}\right), 3.67\left(\mathrm{dd}, 1 \mathrm{H}, J=9.8,3.2 \mathrm{~Hz}, \mathrm{H}_{3}\right.$ ) , $2.89\left(\mathrm{dt}, 1 \mathrm{H}, 10.6,3.5 \mathrm{~Hz}, \mathrm{H}_{2}\right), 0.95\left(\mathrm{~s}, 9 \mathrm{H}, \mathrm{H}_{5}\right), 0.09$ (s, 3H, H4), 0.07 (s, 3H, H4). ${ }^{13} \mathrm{C}$ NMR $\left(100 \mathrm{MHz}, \mathrm{CDCl}_{3}\right) \delta 202.3\left(\mathrm{C}_{1}\right), 138.2\left(\mathrm{C}_{8}\right), 128.5\left(\mathrm{C}_{10}\right), 127.7\left(\mathrm{C}_{9,11}\right), 73.0\left(\mathrm{C}_{7}\right), 66.6\left(\mathrm{C}_{3,3^{\prime}}\right)$, $48.7\left(\mathrm{C}_{2}\right), 26.8\left(\mathrm{C}_{5}\right), 17.7\left(\mathrm{C}_{6}\right),-6.1\left(\mathrm{C}_{4}\right),-6.2\left(\mathrm{C}_{4}\right)$. Anal. Calcd for $\mathrm{C}_{16} \mathrm{H}_{26} \mathrm{O}_{2} \mathrm{Si}: \mathrm{C}, 69.01 ; \mathrm{H}$, 9.41. Found: C, 69.31; H, 9.62\%.

3-Benzylamino-3-tert-butyldimethylsilyl-propan-1,2-diol (24). To a cooled $\left(0^{\circ} \mathrm{C}\right)$ solution of the epoxy alcohol 2 (500 mg, $2.65 \mathrm{mmol}, 1.0$ equiv.) and benzylamine (0.35 mL, $3.19 \mathrm{mmol}, 1.2$ equiv.) in $\mathrm{CH}_{2} \mathrm{Cl}_{2}(12 \mathrm{~mL})$, was slowly added titanium tetra-iso-propoxide $(1.60 \mathrm{~mL}, 5.31 \mathrm{mmol}$, 2.0 equiv.). The solution was stirred for 1 hour and was warmed up to $25-30^{\circ} \mathrm{C}$. The medium was stirred overnight at this temperature and, after cooling down to $0^{\circ} \mathrm{C}$, was carefully quenched with a $10 \%$ aqueous $\mathrm{NaOH}$ solution $(10 \mathrm{~mL})$. After 30 minutes, the mixture was progressively warmed to RT and the titanium salts were filtered off by using a pad of Celite. The salts were washed three times with $\mathrm{CH}_{2} \mathrm{Cl}_{2}$ and filtered off. The combined organic layers were washed successively with a saturated aq. $\mathrm{NH}_{4} \mathrm{Cl}$ solution, then brine, and dried over $\mathrm{Na}_{2} \mathrm{SO}_{4}$. After removal of the solvents and evaporation in vacuo, the crude product was purified by flash chromatography on silica gel (petroleum/AcOEt:80/20), affording the amino-diol $\mathbf{2 4}$ as a colorless oil (595 mg, $2.00 \mathrm{mmol}, 76 \%$ yield). IR (neat) 3460, 3010, 2970, 1240, $850 \mathrm{~cm}^{-1}{ }^{1} \mathrm{H}$ NMR (400 MHz, $\left.\mathrm{CDCl}_{3}\right) \delta 7.27-7.22\left(\mathrm{~m}, 5 \mathrm{H}, \mathrm{H}_{9,10,11}\right), 4.13\left(\mathrm{~d}, 1 \mathrm{H}, J=12.2 \mathrm{~Hz}, \mathrm{H}_{7}\right), 3.90$ (dd, $\left.1 \mathrm{H}, J=11.2,3.6 \mathrm{~Hz}, \mathrm{H}_{1}\right), 3.80\left(\mathrm{bq}, 1 \mathrm{H}, J=3.6 \mathrm{~Hz}, \mathrm{H}_{2}\right), 3.79\left(\mathrm{dd}, 1 \mathrm{H}, J=11.2,3.6 \mathrm{~Hz}, \mathrm{H}_{1}\right.$ ) $), 3.74$ $\left(\mathrm{d}, 1 \mathrm{H}, J=12.2 \mathrm{~Hz}, \mathrm{H}_{7}\right.$ ) $, 2.67\left(\mathrm{dd}, 1 \mathrm{H}, J=3.6,1.6 \mathrm{~Hz}, \mathrm{H}_{3}\right), 0.90\left(\mathrm{~s}, 9 \mathrm{H}, \mathrm{H}_{5}\right), 0.04\left(\mathrm{~s}, 3 \mathrm{H}, \mathrm{H}_{4}\right)$, $0.01\left(\mathrm{~s}, 3 \mathrm{H}, \mathrm{H}_{4}\right) .{ }^{13} \mathrm{C} \mathrm{NMR}\left(100 \mathrm{MHz}, \mathrm{CDCl}_{3}\right) \delta 139.9,128.8,128.7,127.6,73.0,66.9,57.5$, $52.3,27.1,17.3,-5.9,-7.1$.

\section{1-Benzylamino-1-tert-butyldimethylsilyl-3-tert-butyldimethylsilanyloxy-propan-2-ol}

(25).

The silylation of the primary hydroxyl group of the amino-diol 24 (148 $\mathrm{mg}, 0.50 \mathrm{mmol}, 1$ equiv.) was carried out using the procedure described for the preparation of $\mathbf{3 1}$. The amino alcohol 25 was obtained as a colorless oil (198 mg, $0.48 \mathrm{mmol}, 97 \%$ ). IR (neat) 3420, 2900, 1240, $820 \mathrm{~cm}^{-1}$. ${ }^{1} \mathrm{H}$ NMR (400 MHz, $\left.\mathrm{CDCl}_{3}\right) \delta 7.35-7.27\left(\mathrm{~m}, 5 \mathrm{H}, \mathrm{H}_{12,13,14}\right), 4.03\left(\mathrm{~d}, 1 \mathrm{H}, J=12.7 \mathrm{~Hz}, \mathrm{H}_{10}\right), 3.98$ $\left(\mathrm{m}, 1 \mathrm{H}, \mathrm{H}_{2}\right), 3.77\left(\mathrm{~d}, 1 \mathrm{H}, J=12.7 \mathrm{~Hz}, \mathrm{H}_{10}\right), 3.76\left(\mathrm{dd}, 1 \mathrm{H}, J=10.2,8.1 \mathrm{~Hz}, \mathrm{H}_{3}\right), 3.69$ (dd, $1 \mathrm{H}, J$ $\left.=10.2,4.0 \mathrm{~Hz}, \mathrm{H}_{3}{ }^{\prime}\right), 2.49\left(\mathrm{~d}, 1 \mathrm{H}, J=4.1 \mathrm{~Hz}, \mathrm{H}_{1}\right), 0.96\left(\mathrm{~s}, 9 \mathrm{H}, \mathrm{H}_{5}\right), 0.95\left(\mathrm{~s}, 9 \mathrm{H}, \mathrm{H}_{8}\right), 0.13(\mathrm{~s}, 3 \mathrm{H}$, $\left.\mathrm{H}_{7}\right), 0.13\left(\mathrm{~s}, 3 \mathrm{H}, \mathrm{H}_{4}\right), 0.09\left(\mathrm{~s}, 3 \mathrm{H}, \mathrm{H}_{7}\right), 0.08\left(\mathrm{~s}, 3 \mathrm{H}, \mathrm{H}_{4}\right)$ ). ${ }^{13} \mathrm{C} \mathrm{NMR}\left(100 \mathrm{MHz}, \mathrm{CDCl}_{3}\right) \delta 141.0$, $128.5,128.4,127.0,74.6,65.5,55.7,49.0,27.3,26.0,18.4,17.2,-5.1,-5.1,-6.3$. Anal. Calcd for $\mathrm{C}_{22} \mathrm{H}_{43} \mathrm{NO}_{2} \mathrm{Si}_{2}$ : C, 64.49; H, 10.58; N, 3.42. Found: C, 64.38; H, 10.77; N, 3.44\%. 


\section{References}

1. Mulzer, J. In Stereosel. Synth. Ottow, E.; Schoellkopf, K.; Schulz, B.-G., Eds.; Springer: Berlin, 1993; 37.

2. Marié, J.-C.; Courillon, C.; Malacria, M. Eur. J. Org. Chem. 2006, 463.

3. Marié, J.-C.; Courillon, C.; Malacria, M. Tetrahedron 2003, 59, 9759.

4. Marié, J.-C.; Courillon, C.; Malacria, M. Synlett 2002, 553.

5. Padwa, A.; Murphee, S. S. Arkivoc 2006, (iii), 6.

6. Chakraborty, T. K.; Laxman, P. Tetrahedron Lett. 2002, 43, 2645; (b) Wipf, P.; Fritch, P. C. J. Org. Chem. 1994, 59, 4875; (c) Sabitha, G.; Babu, S. R.; Reddy, M. S. K.; Yadav, J. S. Synthesis, 2002, 2254; (d) Sabitha, G.; Babu, R. S.; Rajkumar, M.; Yadav, J. S. Org. Lett., 2002, 4, 343.

7. Marion, F.; Calvet, S.; Marié, J.-C.; Courillon, C.; Malacria, M. Eur. J. Org. Chem. 2006, 453.

8. Gilloir, F.; Malacria, M. Tetrahedron Lett. 1992, 33, 3849.

9. Chakraborty, T. K.; Reddy, G. V. Tetrahedron Lett. 1990, 31, 1335.

10. Chakraborty, T. K.; Reddy, G. V. Tetrahedron Lett. 1991, 32, 679.

11. Regueiro-Ren, A.; Borzilleri, R. M.; Zheng, X.; Kim, S.-H.; Johnson, J. A.; Fairchild, C. R.; Lee, F. Y. F.; Long, B. H.; Vite, G. D. Org. Lett. 2001, 3, 2693.

12. Manis, P. A.; Rathke, M. W. J. Org. Chem. 1980, 45, 4952.

13. Jarvis, B. B.; Nicholas, P. E. J. Org. Chem. 1979, 44, 2951.

14. Jarvis, B. B.; Nicholas, P. E. J. Org. Chem. 1980, 45, 2265.

15. Canas, M.; Poch, M.; Verdaguer, X.; Moyano, A.; Pericas, M. A.; Riera, A. Tetrahedron Lett. 1991, 32, 6931.

16. Le Bideau, F.; Gilloir, F.; Nilsson, Y.; Aubert, C.; Malacria, M. Tetrahedron Lett. 1995, 36, 1641. (b) Le Bideau, F.; Gilloir, F.; Nilsson, Y.; Aubert, C.; Malacria, M. Tetrahedron 1996, 52,7487 . 